\title{
Textos periodísticos sobre salud pública y gestión sanitaria en el diario El País, 2001-2016
}

\section{Journalistic texts on public health and health management in the newspaper El País, 2001-2016}

\author{
María González-García ${ }^{a, f}$, Begoña Cantabranab,e,f, Ana Fernández-Feitoc,f, Alberto Lanad,f, Agustín Hidalgob,e,f \\ a Hospital Universitario Central de Asturias, Oviedo, España \\ b Área de Farmacología, Departamento de Medicina, Universidad de Oviedo, España \\ c Área de Enfermería, Departamento de Medicina, Universidad de Oviedo, España \\ d Área de Medicina y Salud Pública, Departamento de Medicina, Universidad de Oviedo, España \\ e Instituto Universitario de Oncología del Principado de Asturias, Fundación CajAstur, Oviedo, España \\ f Instituto de Investigación Sanitaria de Asturias, Oviedo, España
}

\section{Resumen}

Introducción: la prensa ha adquirido un papel relevante en la formación, conocimiento y expectativas de salud en la sociedad. Objetivo: analizar los temas relacionados con salud pública y gestión sanitaria publicados en el diario El País durante 2001-2016. Métodos: se realizó un estudio documental, con enfoque cuantitativo, seleccionando documentos de los años indicados relacionados con salud pública y gestión sanitaria, contenidos en el cuerpo de los textos periodísticos sobre Medicina y Salud de la colección CONPRE-Oviedo. Resultados: se identificaron 265 artículos sobre salud pública y 326 sobre gestión sanitaria. Los de salud pública se relacionaron con la prevención de la enfermedad y promoción de la salud (30,9\%), epidemiología (25,3\%), drogas ilegales, alcohol y tabaco (25,3\%). En gestión sanitaria fueron macrogestión (61,3\%), mesogestión (27,9\%) y microgestión $(10,7 \%)$. Los artículos fueron preferentemente informativos, de fuente institucional, e incluidos mayoritariamente en la sección de Sociedad. Los textos sobre prevención de la enfermedad y promoción de la salud se basaron en publicaciones científicas. Conclusión: el diario El País incluyó frecuentemente temas relacionados con salud pública y gestión sanitaria, por lo que los textos periodísticos podrían contribuir a la formación de la población y a generar una actitud crítica en salud.

Palabras clave: medios de comunicación de masa; salud pública; gestión sanitaria; educación en salud.

\begin{abstract}
Introduction: the press has acquired a relevant role in health education, knowledge and expectations in society. Objective: to carry out a descriptive analysis of the issues related to public health and health management published in the newspaper El País during 2001-2016. Methods: A documentary study was carried out, with a quantitative approach, selecting documents related to public health and health management, contained in the body of journalistic texts on Medicine and Health of the CONPRE-Oviedo collection. Results: 265 journalistic texts on public health and 365 on health management were identified. Public health was linked to disease prevention and health promotion (30.9\%), epidemiology (25.3\%), illicit drugs, alcohol and tobacco $(25.3 \%)$. The texts on health management focused on macro-management $(61.3 \%)$, meso-management $(27.9 \%)$ and micromanagement (10.7\%). The articles were preferably informative, from an institutional source, and mostly included in the Society section. Texts on disease prevention and health promotion were based on scientific publications. Society is the section where they are most frequently published. Conclusion: the newspaper El País has frequently covered topics related to public health and health management, so journalistic texts could contribute to population education and to the generation of a critical attitude towards health.
\end{abstract}

Keywords: mass media; public health; health management; health education. 


\section{Introducción}

os medios de comunicación han ido adquiriendo un papel relevante en la formación de una imagen del conocimiento y de las expectativas sobre salud de la sociedad (Lobera, 2017; Riobó, 2016). En consecuencia, el espacio dedicado por los medios de comunicación a la información en salud ha crecido en los últimos decenios. Paralelamente, también se ha incrementado la credibilidad de la prensa en materia de salud. La base verídica de los textos periodísticos es cada vez más sólida, ya que se usan más y mejores fuentes para su elaboración. Además, en ello suelen coincidir factores de proximidad, magnitud e implicación social (Martínez Segura, 2006), lo que contribuye aún más a atraer el interés individual y colectivo. Todo ello otorga a la prensa un papel potencialmente relevante en la formación de ciudadanos instruidos, incluida la educación para la salud.

Por otra parte, y dado que la Ley 33/2011 General de Salud Pública reconoce el derecho de los ciudadanos a ser informados, no es infrecuente que las administraciones competentes recurran a los medios de comunicación de masas para informar sobre algunas iniciativas 0 implementar campañas de salud pública (p. ej. de vacunación, antitabaco, etc.). Tampoco es infrecuente que se utilicen los medios como base documental para realizar determinadas evaluaciones (p.ej. del desarrollo científico, del tratamiento dado a la innovación científica, o de la eficacia en la consecución de los fines promovidos en las campañas) (González Silva, 2014; Stead et al., 2019; Wakefield, Loken \& Hornik, 2010; Wakefield, Spittal, Yong, Durkin \& Borland, 2011).

Los medios de comunicación se han erigido en un foro para el debate público de problemas científicos, de acciones de salud pública, de políticas en salud y de gestión sanitaria. En ocasiones, los debates sobre salud desbordan el ámbito de los expertos y se trasladan al espacio público. Este hecho, si bien redunda en la formación crítica de la ciudadanía, puede tener consecuencias negativas derivadas de potenciales contradicciones entre expertos, lo que conduciría al desconcierto entre la población y a la merma del crédito en la práctica sanitaria (Tuells, 2016).

Actualmente se acepta que la prensa tiene un papel crucial en la formación de opiniones y en el modelado de diferentes conductas de salud (Odone, Tramutola, Morgado \& Signorelli, 2018). Existen estudios sobre el tratamiento que la prensa otorga a los descubrimientos sobre el cáncer (González Silva, 2014), las epidemias (Amo Valero, Coiras López, Díaz Franco \& Pérez Olmeda, 2017; Camacho Markina, 2009), las vacunas (Catalán-Matamoros \& Peñafiel-Saiz, 2019a; CatalánMatamoros \& Peñafiel-Saiz, 2019b; Martínez Martínez, Tuells \& Colmenar-Jarillo, 2015; Moreno Castro, 2017), los cuidados paliativos (Carrasco et al., 2017) o la demografía sanitaria (Rodríguez-Arrastia, Moreno-Castro \& Ropero-Padilla, 2019). También se ha documentado el impacto de la prensa sobre la adquisición de conductas saludables, como la reducción del consumo de tabaco y de alcohol (Robinson et al., 2014; Wakefield et al., 2010; Wakefield et al., 2011) o la adquisición de hábitos para el control del dolor lumbar (Suman et al., 2020). En definitiva, parece evidente que la prensa desempeña un papel relevante en la salud y que los textos periodísticos pueden ser tratados como base documental sobre la que analizar diferentes aspectos de la comunicación en salud, incluidos sus propios motivos (González-García, Cantabrana \& Hidalgo, 2020; Revuelta, 2006), el impacto de políticas de comunicación (Jiménez Sarmiento, 2020) o el tratamiento informativo de determinados problemas de salud en un ámbito concreto (Rodríguez García \& Ramos Martínez, 2017).

El objetivo de este estudio fue analizar los textos periodísticos sobre salud pública y gestión sanitaria aparecidos en el diario El País (España) en el periodo 2001-2016.

\section{Métodos}

La fuente de los textos periodísticos utilizados ha sido la COlección de Noticias de PREnsa de la Universidad de Oviedo (CONPRE-Oviedo), que está compuesta por 7.827 textos publicados entre el 1 de enero de 2001 y el 30 de diciembre de 2016 en el diario El País (España).

Los criterios de selección de los textos periodísticos para crear la colección fueron los siguientes: a) el título debe aludir a términos de interés para la colección (ciencia y tecnología, medicina y salud, medicamentos, educación universitaria, sociología, terminología, humor y comunicación de los pacientes mediante cartas al editor); b) tener un autor identificable; c) estar basado en hechos reales; d) tener entidad narrativa; y e) contener una historia completa. La distribución en apartados y subapartados se llevó a cabo por, tres observadores independientes ( $\mathrm{AH}, \mathrm{MGG}, \mathrm{BC})$, consensuando la decisión en casos de dudas.

De todos los textos de la colección CONPRE-Oviedo, 2.001 textos pertenecen a la categoría de Medicina y 
Salud (González, Cantabrana \& Hidalgo, 2020). Sobre ellos, se realizó un estudio documental, con enfoque cuantitativo. Para ello, se identificaron los textos que por su contenido pudieron ser clasificados bajo los rótulos de salud pública y gestión sanitaria. Esta selección la realizaron también dos investigadores independientes (AH y MGG).

Para la clasificación se tuvieron en cuenta los siguientes criterios: orientación del artículo según su contenido, propósito del mismo, relación con apartados tradicionales de la salud pública (información sobre problemas de salud, educación para la salud, cribados poblacionales...) y también de la gestión sanitaria. La evaluación de los textos, según los criterios antedichos, nos ha permitido extraer información relevante para realizar los siguientes análisis: a) distribución anual de las noticias, calculando la media anual de textos; b) distribución en diferentes apartados según la temática abordada; c) asignación a las categorías de macrogestión (o política sanitaria), mesogestión (o gestión de centros) y microgestión (o gestión clínica) en el caso de la gestión sanitaria; d) distribución según las diferentes categorías periodísticas (enfoque, género periodístico, fuente de referencia, sección del diario y presencia o no de imágenes acompañantes); y e) distribución en los diferentes objetivos de comunicación. Con los datos obtenidos se realizó una distribución de frecuencias mostrando los datos como media y rango, en valores absolutos y/o relativos.

\section{Resultados}

El estudio, realizado sobre 591 textos periodísticos, muestra la presencia continua de textos sobre salud pública y más incidental sobre gestión sanitaria, a lo largo de los 16 años del periodo de análisis. En salud pública se han centrado, de forma sucesiva, en los cribados poblacionales, la prevención secundaria y las enfermedades crónicas, mientras que la macrogestión acumula el mayor número de textos sobre gestión sanitaria. Los textos sobre salud pública fueron mayoritariamente informativos y los de gestión sanitaria de opinión. En ambos casos se publicaron de forma preferente en la sección de Sociedad. En los años finales del estudio la sociedad civil adquiere protagonismo informativo en temas de salud.

\section{Número y distribución de las noticias}

Durante el periodo del estudio se identificaron 591 noticias, distribuidas en salud pública ( $n=265 ; 44,8 \%$ ) y gestión sanitaria $(n=326 ; 55,2 \%)$. La media global fue de 36,9 textos por cada año, de los cuales 16,6 (rango: 8-26) fueron de salud pública y 20,4 (rango: 1-62) de gestión sanitaria. Como muestra la figura 1, la evolución anual de los textos sobre salud pública fue constante, mientras que en los de gestión sanitaria fue más variable, produciéndose un incremento sustancial durante el periodo 2012-2015, con un pico máximo en el año 2012 (n=62) (Figura 1).

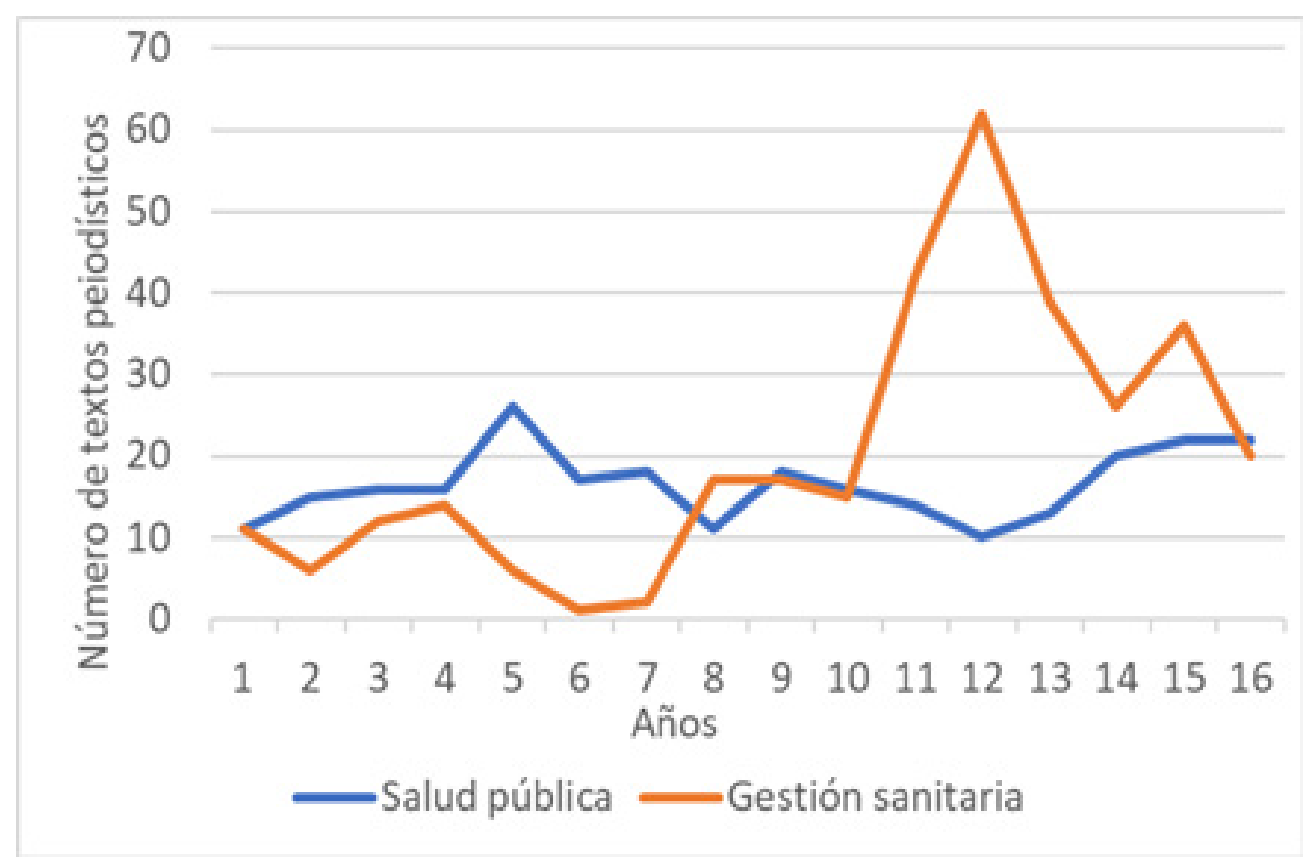

Figura 1. Evolución temporal (años 2001 a 2016) del número de textos periodísticos sobre salud pública y gestión sanitaria en el diario El País. 


\section{Textos sobre salud pública}

Los textos incluidos bajo el epígrafe salud pública tuvieron que ver, en orden decreciente según su frecuencia, con la prevención de la enfermedad y promoción de la salud, la epidemiología, las drogas ilegales, los factores sociales, el alcohol y el tabaco, la sanidad ambiental, y la seguridad alimentaria (tabla 1). En los textos periodísticos de este apartado se diferenciaron tres etapas. La primera (20012006), en la que los cribados poblacionales y la prevención secundaria fueron los protagonistas; la segunda (2007-
2013), relacionada con la prevención de la obesidad, la dieta saludable y las medidas políticas; y la tercera (20132016), vinculada a enfermedades crónicas, promoción de la salud a través de hábitos saludables, dieta y ejercicio físico. Se detectaron dos picos incidentales, uno en 2005, relacionado con la prevención del $\mathrm{VIH}$, el uso de métodos anticonceptivos de barrera y las actitudes sociales; y otro en el año 2015, referido al efecto cancerígeno de la carne roja y productos procesados. Los textos relacionados con cáncer y obesidad también siguieron esta evolución (Tabla 1).

Tabla 1. Características de los textos periodísticos de los diferentes apartados de salud pública y gestión sanitaria.

\begin{tabular}{|l|l|l|l|l|l|l|l|l|l|l|}
\hline \multicolumn{9}{|c}{ Salud Pública } & \multicolumn{3}{c|}{ Gestión Sanitaria } & \\
\hline & $\begin{array}{l}\text { Prevención, } \\
\text { Promoción* } \\
(82 ; 13,9 \%)\end{array}$ & $\begin{array}{l}\text { Epidemiología } \\
(67 ; 11,3 \%)\end{array}$ & $\begin{array}{l}\text { Drogas } \\
\text { ilegales } \\
(35 ; 5,9 \%)\end{array}$ & $\begin{array}{l}\text { Alcohol } \\
(16 ; 2,7 \%)\end{array}$ & $\begin{array}{l}\text { Tabaco } \\
(16 ; 2,7 \%)\end{array}$ & $\begin{array}{l}\text { Otras } \\
(49 ; 8,3 \%)\end{array}$ & $\begin{array}{l}\text { Meso- } \\
\text { gestión } \\
(91 ; 15,4 \%)\end{array}$ & $\begin{array}{l}\text { Micro } \\
\text { gestión } \\
(35 ; 5,9 \%)\end{array}$ & $\begin{array}{l}\text { Micro } \\
\text { gestión } \\
(35 ; 5,9 \%)\end{array}$ & $\begin{array}{l}\text { Total } \\
(591 ; 100 \%)\end{array}$ \\
\hline Enfoque \% & & & & & & & & & & \\
\hline Científico & 58,5 & 80,6 & 48,6 & 43,8 & 25 & 16,30 & 0 & 2 & 5,7 & 24,4 \\
\hline Sociocultural & 41,4 & 19,4 & 51,4 & 56,2 & 75 & 83,7 & 100 & 98 & 94,3 & 75,6 \\
\hline Género \% & & & & & & & & & & \\
\hline Informativo & 51,2 & 64,2 & 68,6 & 50,0 & 56,3 & 65,3 & 78 & 54,5 & 54,4 & 60,4 \\
\hline Interpretativo & 28,1 & 26,9 & 31,4 & 25,0 & 37,5 & 18,4 & 10 & 20,5 & 22,8 & 21,8 \\
\hline Opinión & 20,7 & 8,9 & 0 & 25,0 & 6,2 & 16,3 & 12 & 25 & 22,8 & 17,8 \\
\hline Fuente \% & & & & & & & & & & \\
\hline Experto & 32,9 & 34,3 & 5,7 & 18,7 & 12,5 & 20,4 & 15,4 & 24,5 & 48,6 & 24,9 \\
\hline Institucional & 24,4 & 43,3 & 45,7 & 37,5 & 62,5 & 26,5 & 36,3 & 44,5 & 14,2 & 37,4 \\
\hline $\begin{array}{l}\text { Rev } \\
\text { Científica }\end{array}$ & 15,9 & 16,4 & 5,7 & 12,5 & 6,3 & 2 & 0 & 0 & 2,9 & 5,2 \\
\hline Sección \% & & & & & & & & & & \\
\hline Sociedad & 69,5 & 68,7 & 60 & 68,8 & 68,8 & 71,4 & 75,8 & 68 & 54,3 & 68,5 \\
\hline Otros & 30,5 & 31,3 & 40 & 31,2 & 31,2 & 28,6 & 24,2 & 32 & 45,7 & 31,5 \\
\hline Infografías \% & & & & & & & & & & \\
\hline Si & 11,0 & 35,8 & 40,0 & 31,3 & 25,0 & 12,2 & 9,9 & 21 & 14,3 & 20 \\
\hline No & 89,0 & 64,2 & 60,0 & 68,8 & 75,0 & 87,8 & 90,1 & 79 & 85,7 & 80 \\
\hline
\end{tabular}




\section{Textos sobre gestión sanitaria}

Los textos relacionados con gestión sanitaria se distribuyeron en tres apartados: macrogestión, mesogestión y microgestión, ordenados de mayor a menor según frecuencia. Hasta el año 2010, el número de noticias que formaron la colección fue inferior a 20 noticias/año, centradas en el traspaso sanitario a las Comunidades Autónomas, la Ley de Cohesión y Calidad del Sistema Nacional de Salud, la desigualdad interterritorial, así como las repercusiones de dicha descentralización y la modificación del sistema de financiación.

A partir de 2010 se observó una tendencia a informar sobre el gasto público en salud, y la disminución de la contratación, además del atraso de pagos de material sanitario y medicamentos motivados por la crisis económica que se inició en el año 2008 y que provocó recortes en la mayor parte de los apartados de la economía española. Los textos sobre macrogestión y mesogestión se incrementaron de manera importante alcanzando el máximo en 2012 (figura 2). Casi la totalidad de los textos periodísticos durante este periodo se relacionaron con el gasto sanitario, concretamente con los recortes sanitarios, el copago, la privatización y el Real Decreto-ley 16/2012, de 20 de abril, de medidas urgentes para garantizar la sostenibilidad del Sistema Nacional de Salud y mejorar la calidad y seguridad de sus prestaciones (Figura 2).

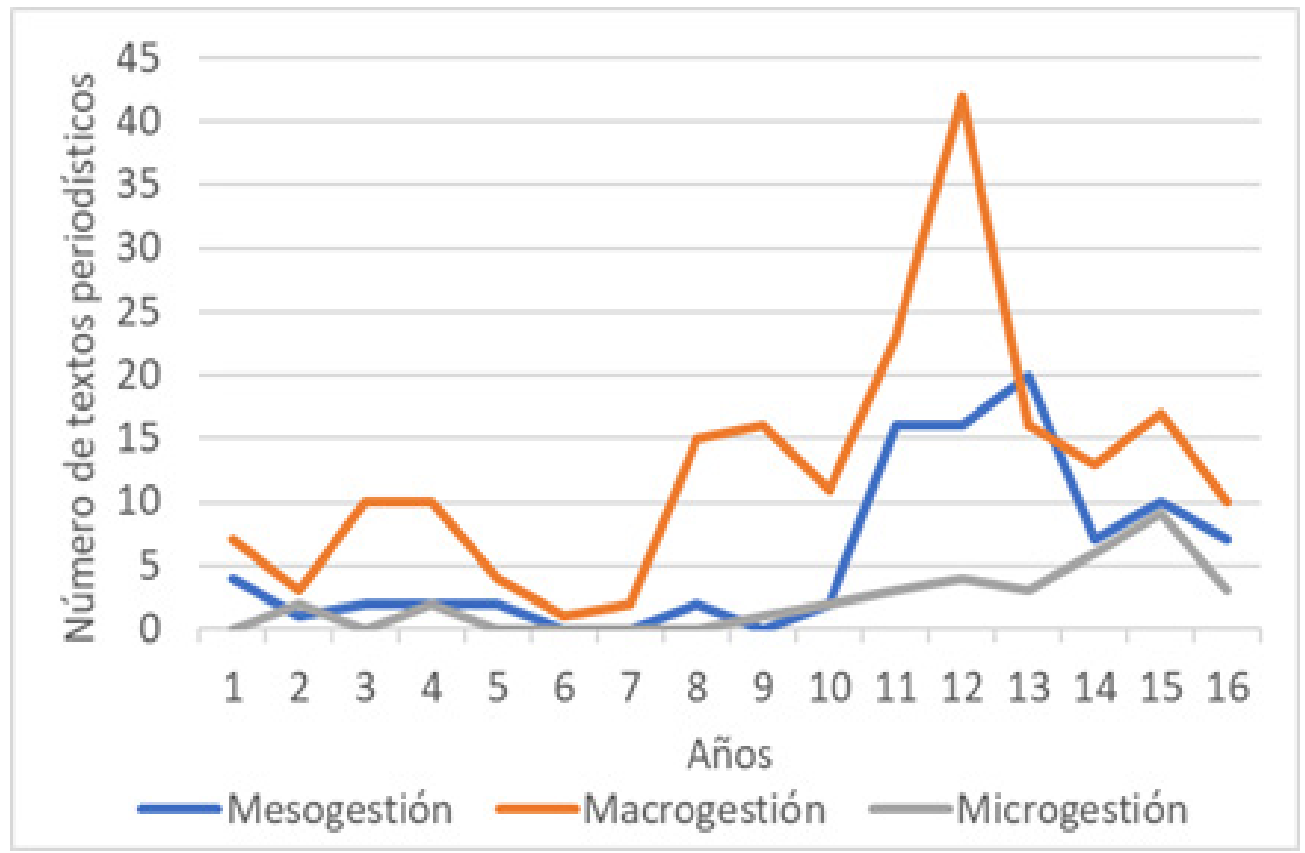

Figura 2. Evolución temporal (años 2001 a 2016) del número de textos periodísticos sobre mesogestión, macrogestión y microgestión en el diario El País. 
Análisis de características de los textos periodísticos

En la tabla 1 se muestra la distribución de textos según el género periodístico, la sección del diario, su enfoque, la fuente y la presencia o no de imágenes de acompañamiento a los textos. En relación con el género periodístico, el informativo fue el más frecuente en todas las secciones, alcanzando entre un 50 y un $80 \%$ del total. El género interpretativo, a través de los reportajes, fue más frecuente en los artículos de salud pública, mientras que en la gestión sanitaria fueron los artículos de opinión (tabla 1). De 591 artículos que componen la muestra, más de la mitad $(68 \%)$ se publicaron en la sección de Sociedad. El enfoque de los textos estuvo claramente diferenciado: en salud pública, la mayoría tiene un enfoque científico, mientras que casi el $100 \%$ de los relacionados con gestión sanitaria tienen un enfoque sociocultural.

Entre las fuentes de información utilizadas para la elaboración de los textos periodísticos, la de tipo institucional (hospitales, políticos, ministerio, plan nacional sobre drogas, etc.) fue la más utilizada. Por el contrario, en apartados como prevención de la enfermedad y promoción de la salud, epidemiología y microgestión, la fuente más habitual fue la opinión de expertos, con una contribución no desdeñable de las revistas científicas. La sociedad civil (ciudadanos, sindicatos, asociaciones de pacientes, organizaciones sin ánimo de lucro) fue la fuente más frecuente en mesogestión (Tabla 1). Si observamos la evolución de las fuentes consultadas a lo largo de los 16 años, constatamos que las revistas científicas fueron una referencia constante, mientras que el resto de las fuentes presentaron oscilaciones. A partir del 2011, la sociedad civil superó la veintena de textos, manteniéndose constante hasta el final del periodo estudiado.

\section{Finalidad de los textos periodísticos}

En cuanto a la finalidad de los textos periodísticos, el objetivo más frecuente fue la función educativa $(n=388$, $65,6 \%$ ), seguido de la inducción al cambio actitudinal o comportamental ( $n=91,15,4 \%)$. Los objetivos de crear confianza $(n=59)$ o inducir a la participación $(n=53)$ estuvieron presentes en aproximadamente el $10 \%$ de los textos. Algunos ejemplos de estos textos se muestran en la tabla 2. Si relacionamos la fuente consultada con el objetivo de comunicación que se quiere alcanzar, se observó que la sociedad civil tuvo como objetivo principal inducir a la participación del receptor del mensaje; las fuentes institucionales y expertos tuvieron como objetivo la función educadora y de creación de confianza; y las revistas científicas fueron invocadas con objetivos tanto informativos como de inducción al cambio (Figura 3). Ejemplos paradigmáticos pueden ser el papel adoptado ante la privatización de la sanidad en la Comunidad de Madrid 0 ante la disponibilidad de oseltamivir para el tratamiento de la hepatitis $\mathrm{C}$.

Tabla 2. Ejemplos que reflejan la finalidad de los textos periodísticos sobre salud pública y gestión sanitaria.

\begin{tabular}{|l|l|}
\hline \multicolumn{1}{|c|}{ Finalidad } & \multicolumn{1}{c|}{ Ejemplo de texto } \\
\hline Educación & "La sanidad se vuelve autonómica." Milagros Pérez Oliva, \\
& $\begin{array}{l}\text { 2004. } \\
\text { "Una hora de ejercicio al día contrarresta el efecto de ocho } \\
\\
\text { quieto." Nuño Domínguez, 2015. }\end{array}$ \\
\hline Creación de confianza & "El copago sanitario tiene alternativas." Antonio González, \\
& $\begin{array}{l}2010 . \\
\text { "El gasto en sanidad y educación subió en 2014, por primera } \\
\text { vez en la crisis." Jesús Sérvulo González, 2015. }\end{array}$ \\
\hline Inducción a la participación & "Los enfermos crónicos exigen participar en las decisiones \\
& $\begin{array}{l}\text { del sistema de salud." Emilio de Benito, 2005. } \\
\text { "Los recortes de la Generalitat incendian la sanidad } \\
\text { catalana." Oriol Güel, 2011. }\end{array}$ \\
\hline Inducción al cambio & "El consejo de Europa advierte a España del impacto social \\
& $\begin{array}{l}\text { de los recortes." Esperanza Escribano, 2013. } \\
\text { "Cómo cambiar el sistema nacional de salud." Rafael } \\
\text { Bengoa, 2014. }\end{array}$ \\
\hline
\end{tabular}




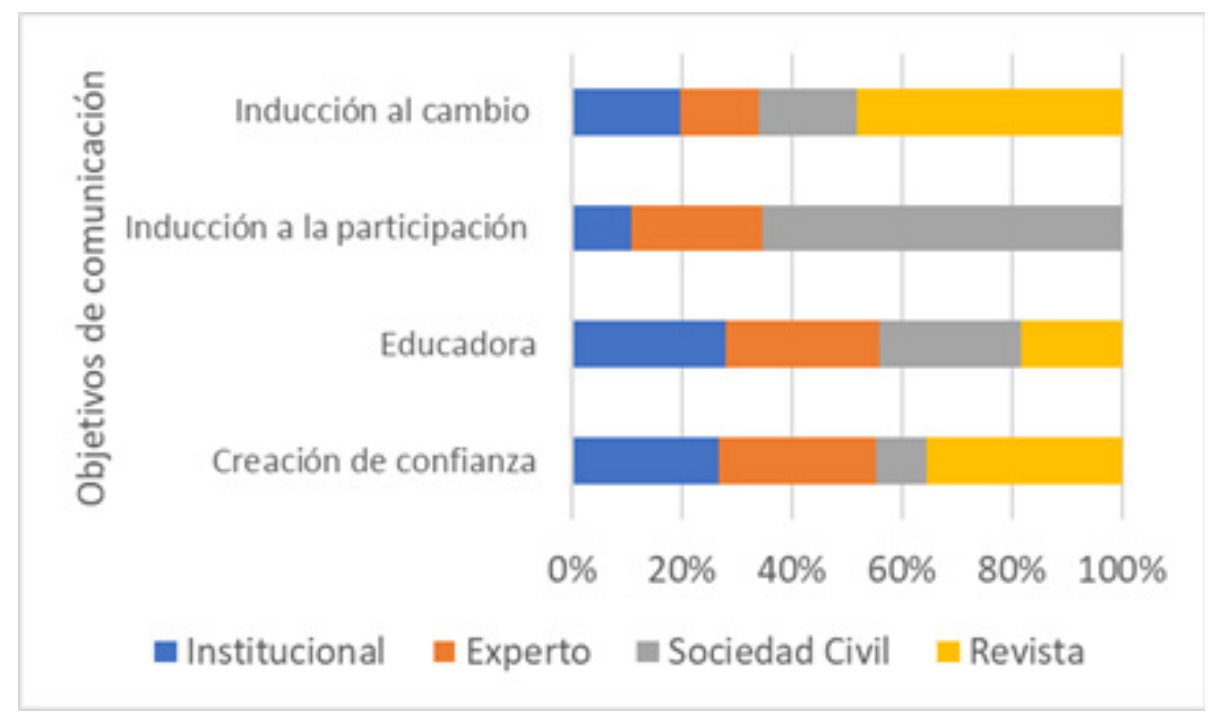

Figura 3. Distribución de los objetivos de comunicación mediante textos periodísticos según la fuente documental en que se basa la información

\section{Discusión}

En líneas generales, la información de los medios de comunicación conjuga componentes sociales, políticos y económicos, lo que les confiere no solo interés público sino también una dimensión sociológica que puede ser observada y analizada de forma científica. Además, los diarios pueden ser útiles para la iniciación de una cultura médica tanto en los estudiantes de ciencias de la salud como en la población en general, a través de la divulgación científica (Cantabrana, Diez \& Hidalgo, 2015) de temas médicos como de la apropiación de terminología médica derivada de los artículos periodísticos (Cantabrana, Diez, Bordallo, Sánchez \& Hidalgo, 2013). Esto es posible porque la información en salud es frecuente en la prensa (González et al, 2020; González-García et al, 2020; Revuelta, 2006) y ha ido adquiriendo una calidad creciente. Un ejemplo es la información sobre diferentes aspectos de salud pública y gestión sanitaria, que supone el $29,5 \%$ de las noticias sobre Medicina y Salud que contiene la base de datos CONPRE-Oviedo. Estas están distribuidas a lo largo de todo el período de estudio y, como ocurre en otras bases de datos (Revuelta, 2006), hay temas que tienen un tratamiento continuo y otros de forma más incidental, como el Ébola, el Zika o la Gripe A.

Los temas relacionados con la salud pública sugieren que una amplia variedad de ellos ha alcanzado relevancia informativa $y$, en consecuencia, pueden contribuir a la formación de una cultura en salud. Entre ellos se encuentran temas emergentes tales como la prevención de enfermedades, los cribados poblacionales, la salud ambiental, las drogas ilegales, la promoción de la salud y los hábitos alimentarios. Todo ello corrobora el papel atribuido por otros autores a la prensa en la educación en salud de la ciudadanía (Camacho Markina, 2009; Catalán-Matamoros \& Peñafiel-Saiz, 2019 a,b; Martínez et al., 2015; Moreno Castro, 2017; Odone et al., 2018; Stead et al., 2019; Wakefield et al., 2010;2011;), que puede haber contribuido al aumento de la expectativa (European Commission, 2015) y de la calidad de vida (Eurostat, 2020).

Es importante resaltar que el $25 \%$ de los textos periodísticos estaban relacionadas con las drogas ilegales. A lo largo de los 16 años, se nos muestran los cambios de comportamiento de la población ante el consumo de sustancias ilegales, así como el perfil del consumidor y las sustancias más consumidas, adquiriendo mayor noticiabilidad la cocaína que la heroína a partir de 2006 y las drogas de diseño a partir de 2012. El narcotráfico y la actuación política son noticias que también se reflejaron en el período de análisis. Las noticias relacionadas con el tabaco nos informan sobre las repercusiones que tiene en la salud, así como las medidas de prevención, las repercusiones del tabaco sobre la economía y la implantación de la ley antitabaco.

En el campo de la gestión sanitaria destacan temas 
como la descentralización del sistema público de salud y se comienza a poner en duda la sostenibilidad del Sistema Nacional de Salud público y universal y su repercusión en la demografía sanitaria, el aumento de la demanda sanitaria por los ciudadanos, así como el cuestionamiento de algunas políticas sobre el copago. Todo ello, acentuado por la crisis económica y la promulgación del Real Decreto-ley 16/2012, de medidas urgentes para garantizar la sostenibilidad del Sistema Nacional de Salud y mejorar la calidad y seguridad de sus prestaciones, condicionó la modificación del sistema de financiación y la precariedad laboral de las profesiones sanitarias a la vez que promovió el ahorro sanitario mediante modificaciones en la prestación farmacéutica (introdujo el copago farmacéutico) y de recursos materiales y humanos. Durante el periodo de estudio también hemos asistido al empoderamiento social y a su organización hasta constituirse en un elemento de decisión de las políticas en salud.

Un elemento que otorga relevancia a los textos periodísticos sobre problemas de salud es que aparecen preferentemente en la sección de Sociedad, la de mayor visibilidad en la prensa (Aiestaran, Camacho \& Ronco, 2014; Revuelta, 2006; Ronco López, Peñafiel \& Echegaray, 2014), se suelen acompañar de imágenes de apoyo en el $20 \%$ de los casos, un aspecto relevante para el éxito de la comunicación según algunos autores (Catalán-Matamoros Peñafiel-Saiz, 2019 a,b). Además, aunque la fuente principal de información que sustenta el texto es de origen institucional, como han descrito otros autores (Aiestaran et al., 2014; Revuelta, 2006), es relevante el hecho de que las noticias sobre salud pública se basen en textos científicos porque refuerza su calidad y la adecuada contribución a la educación en salud de la población. También es relevante el recurso a expertos y a revistas científicas cuando se trata de textos relacionados con promoción de la salud y con difundir patrones de prevención de la enfermedad (Gøtzsche \& Nielsen, 2011). Por ello, y de acuerdo con otros autores (Bou-Karroum et al., 2017; Wakefield et al., 2010; 2011; Yadav \& Kobayashi, 2015) consideramos adecuado recurrir a los medios de comunicación, entre ellos la prensa, en las campañas de salud.

Dado que el período de estudio incluye 16 años, es posible observar la creciente influencia de la sociedad civil organizada en asociaciones de pacientes, etc., algo que sugiere un empoderamiento paulatino que ahora es pleno e informado. A ello puede haber contribuido el hecho de que el objetivo preferente de los textos periodísticos tenga, mayoritariamente, función educadora, seguido de la inducción al cambio de actitud a nivel individual y colectivo, lo que otorga a la ciudadanía un papel en la toma de decisiones relacionadas con la salud (López Cerezo, 2018; Weishaar et al., 2016).

\section{Conclusiones}

Del estudio realizado se desprende que los textos periodísticos publicados en el diario El País en el periodo 2001-2016:

1. Incluyeron frecuentemente temas relacionados con salud pública $(44,8 \%)$ y gestión sanitaria $(55,2 \%)$.

2. Se publicaron mayoritariamente en la sección de Sociedad; incluyeron imágenes en el $20 \%$ de los casos; se basaron en fuentes institucionales; y fue frecuente la alusión a artículos científicos como refrendo de la información, particularmente en los textos de promoción de la salud y prevención de enfermedades.

3. Se detectaron dos patrones en el tratamiento de los problemas de salud: a) un tratamiento continuo a lo largo del periodo de estudio (p.ej. cáncer, cribados poblacionales, drogas ilegales, etc.), y b) una presencia puntual de problemas de salud de alta incidencia en períodos concretos y limitados en el tiempo (p.ej. infecciones por virus del Ébola, la Gripe A, el Zika).

4. La repercusión de la crisis económica de 2008 en la atención sanitaria puede ser analizada a partir de los textos referidos a la sostenibilidad del Sistema Nacional de Salud con privatizaciones y reducciones de plantillas y financiación de prestaciones sanitarias, que pueden explicar los problemas asistenciales detectados en situaciones de crisis sanitarias.

5. La ciudadanía se ha ido implicando de forma creciente en las políticas sanitarias hasta alcanzar un empoderamiento pleno e informado.

Por todo ello, consideramos que los textos periodísticos juegan un papel relevante en la creación de una actitud informada en temas de salud y pueden favorecer a la educación para la salud y la participación ciudadana en programas institucionales de prevención de enfermedades.

\section{Contribuciones de los autores}

Los autores participaron igualmente en la elaboración del manuscrito y aprobaron la versión final presentada.

\section{Financiación}

Esta investigación no recibió financiación.

\section{Declaración de disponibilidad de datos}

Los datos presentados en este estudio pueden ser solicitados al autor de correspondencia.

\section{Conflicto de interés}

Los autores declaran que no hay conflicto de interés. 


\section{Referencias bibliográficas}

Amo Valero, J. del., Coiras López, M. T., Díaz Franco, A., \& Pérez Olmeda, M. T. (2017). La investigación contra la gran epidemia del siglo XX. Madrid, España: Instituto de Salud Carlos III. Libros de la Catarata.

Aiestaran, A., Camacho, I., \& Ronco, M. (2014). La información sobre salud y medicina en los diarios de la Comunidad Autónoma Vasca y Navarra. Ámbitos, 26, 1-19.

Bou-Karroum, L., El-Jardali, F., Hemadi, N., Faraj, Y., Ojha, U., Shahrour, M., ... Akl, E. A. (2017). Using media to impact health policy-making: an integrative systematic review. Implementation Science: IS, 12(1), 52. doi: 10.1186/s13012-017-0581-0

Camacho Markina, I. (2009). La 'gripe A', en la prensa española. Revista Latina de Comunicación Social, 64, 827-843. doi: 10.4185/RLCS-64-2009865-827-843

Cantabrana, B., Diez, B., Bordallo, J., Sánchez, M., \& Hidalgo, A. (2013). Apropiación de terminología médica por estudiantes de primer curso del grado en medicina a través de prensa diaria. Revista de la Fundación Educación Médica,16(3),145-51. doi: 10.4321/s2014-98322013000300005

Cantabrana, B., Diez, B., Hidalgo, A. (2015). Percepción por los estudiantes de la divulgación científica en la prensa y de su contribución a la cultura científica. Revista de la Fundación Educación Médica, 18(1), 47-53. doi: 10.4321/S2014-98322015000100008

Carrasco, J. M., García, M., Navas, A., Olza, I., GómezBaceiredo, B., Pujol, F., Garralda, E., \& Centeno, C. (2017). What does the media say about palliative care? A descriptive study of news coverage in written media in Spain. PloS One, 12(10), e0184806. doi: 10.1371/journal.pone.0184806

Catalán-Matamoros, D., \& Peñafiel-Saiz, C. (2019a). A visual content analysis of vaccine coverage in the print media. Human Vaccines \& Immunotherapeutics, 15,2453-2459. doi: 10.1080/21645515.2019.1589289

Catalán-Matamoros, D., \& Peñafiel-Saiz, C. (2019b). How is communication of vaccines in traditional media: a systematic review. Perspectives in Public Health, 139(1), 34-43. doi: 10.1177/1757913918780142

European Commission. (2015). Short Analytical web note 3/2015. Demography report. Luxemburgo: Unión Europea. Recuperado de. https://ec.europa. eu/eurostat/documents/3217494/6917833/KEBM-15-003-EN-N.pdf/76dac490-9176-47bc-80d9029e1d967af6

Eurostat. (2020). Your key to European statistics. Number of healthy years of life: countries compared. Luxemburgo: Unión Europea. Recuperado de https://ec.europa.eu/eurostat/en/web/ products-eurostat-news/-/EDN-20200407-1

González, M., Cantabrana, B. \& Hidalgo, A. (2020). Ciencia, medicina y medicamentos. Un estudio del periodo 2001-2016. Revista de la Fundación Educación Médica, 23(1), 17-24. doi: 10.33588/ fem.231.1034

González-García, M., Cantabrana, B. \& Hidalgo, A (2020). Noticias sobre medicina y salud en un diario de difusión nacional. Potencial utilidad educativa en estudiantes de ciencias de la salud. Revista de Medicina y Cine, 16(3), 223-234. https://doi. org:10.14201/mc2020163223234.

González Silva, M. (2014). Genes de papel: genética, retórica y periodismo en el diario "El País" (1976-2006). Madrid, España: Consejo Superior de Investigaciones Científicas.

Gøtzsche, P. C., \& Nielsen, M. (2011). Screening for breast cancer with mammography. The Cochrane Database of Systematic Reviews, (1), CD001877. doi: 10.1002/14651858.CD001877.pub4.

Jiménez Sarmiento, M. (2020). Análisis de la comunicación de la resitencia a antibióticos en la prensa digital española. Efecto de la aprobación del Plan Nacional de Resistencia a Antibióticos. Revista Española de Comunicación en Salud, 11(1), 65-70. doi: https:// doi.org/10.20318/recs.2020.4887.

Lobera, J. (2017). Percepción social de la ciencia y la tecnología 2016. Madrid, España: Fundación Española para la Ciencia y la Tecnología.

López Cerezo, JA. (2018). La confianza en la sociedad del riesgo. Barcelona, España: Sello Editorial.

Martínez Martínez, P., Tuells, J., \& Colmenar-Jarillo, G. (2015). La tardía emergencia mediática de la vacuna contra la viruela, cobertura de noticias en prensa española (1999-2004). Revista Española de Quimioterapia, 28(3), 125-131. 
Martínez Segura, P. (2006). Crisis sanitaria y medios de comunicación. Revista de Administración Sanitaria, 4(3), 437-448.

Moreno Castro, C. (Ed.). (2017). Campañas institucionales en salud pública: el caso de la vacuna contra el virus VPH. Madrid, España: Dextra editorial.

Odone, A., Tramutola, V., Morgado, M., \& Signorelli, C. (2018). Immunization and media coverage in Italy: an eleven-year analysis (2007-17). Human Vaccines \& Immunotherapeutics, 14, 2533-2536. doi: 10.1080/21645515.2018.1486156

Real Decreto-ley 16/2012, de 20 de abril, de medidas urgentes para garantizar la sostenibilidad del Sistema Nacional de Salud y mejorar la calidad y seguridad de sus prestaciones. Boletín Oficial del Estado, 98, 31278- 31312. Recuperado de https://www.boe.es/eli/ es/rd//2012/04/20/16

Revuelta, G. (2006). Salud y medios de comunicación en España. Gaceta Sanitaria, 20(S1), 203-208. doi: $10.1157 / 13086045$

Riobó, P. (2016). Citas y referencias bibliográficas en publicaciones de salud. Revista Española de Comunicación en Salud, 7(S1), S133-S138. doi: 10.20318/recs.2016.3132

Robinson, M. N., Tansil, K. A., Elder, R. W., Soler, R. E., Labre, M. P., Mercer, S. L., ... Rimer, B.K., Community Preventive Services Task Force (2014). Mass media health communication campaigns combined with health-related product distribution: a community guide systematic review. American Journal of Preventive Medicine, 47(3), 360-371. doi: 10.1016/j. amepre.2014.05.034

Rodríguez-Arrastia, M., Moreno-Castro, C., \& RoperoPadilla, C. (2019). Estudio de las noticias publicadas en prensa del éxodo de enfermeras españolas al Reino Unido. Gaceta Sanitaria, 33(5), 450-454. doi: 10.1016/j.gaceta.2018.04.010

Rodríguez García, M., \& Ramos Martínez, Á. (2017). La información sobre el virus Zika en la prensa local de Almería $=$ Information about Zika virus in the local press of Almería. Revista Española de Comunicación en Salud, 8(1), 29-37. doi: http:// dx.doi.org/10.20318/recs.2017.3604

Ronco López, M., Peñafiel, C., \& Echegaray, L. (2014).
El periodismo de salud en la prensa española (20002010). Aproximación a los estudios documentales existentes. Documentación de las Ciencias de la Información, 37, 267-304. doi: 10.5209/ revDCIN.2014.v37.46827

Suman, A., Armijo-Olivo, S., Deshpande, S., MariettaVasquez, J., Dennett, L., Miciak, M., ... Gross, D. P. (2020). A systematic review of the effectiveness of mass media campaigns for the management of low back pain. Disability and Rehabilitation, 1-29. doi: 10.1080/09638288.2020.1743777

Stead, M., Angus, K., Langley, T., Katikireddi, S. V., Hinds, K., Hilton, S., ... Bauld, L. (2019). Mass media to communicate public health messages in six health topic areas: a systematic review and other reviews of the evidence. NIHR Journals Library, (7)8. doi:10.3310/phr07080

Tuells, J. (2016). Controversias sobre vacunas en España, una oportunidad para la vacunología social. Gaceta Sanitaria, 30(1), 1-3. doi: 10.1016/j. gaceta.2015.10.006

Wakefield, M. A., Loken, B., \& Hornik, R. C. (2010). Use of mass media campaigns to change health behaviour. Lancet, 376(9748), 1261-1271. doi: 10.1016/S01406736(10)60809-4

Wakefield, M. A., Spittal, M. J., Yong, H. H., Durkin, S. J., \& Borland, R. (2011). Effects of mass media campaign exposure intensity and durability on quit attempts in a population-based cohort study. Health Education Research, 26(6), 988-997. doi: 10.1093/her/cyr054

Weishaar, H., Dorfman, L., Freudenberg, N., Hawkins, B., Smith, K., Razum, O., \& Hilton, S. (2016). Why media representations of corporations matter for public health policy: a scoping review. BMC Public Health, 16(899), 1-11. doi: 10.1186/s12889-0163594-8

Yadav, R. P., \& Kobayashi, M. (2015). Asystematic review: effectiveness of mass media campaigns for reducing alcohol-impaired driving and alcohol-related crashes. BMC Public Health, 15(857), 1-17. doi: 10.1186/ s12889-015-2088-4 